\title{
Veech surfaces and simple closed curves
}

\author{
Max Forester, Robert Tang, and Jing Tao
}

\begin{abstract}
We study the SL $(2, \mathbb{R})$-infimal lengths of simple closed curves on half-translation surfaces. Our main result is a characterization of Veech surfaces in terms of these lengths.

We also revisit the "no small virtual triangles" theorem of Smillie and Weiss and establish the following dichotomy: the virtual triangle area spectrum of a half-translation surface either has a gap above zero or is dense in a neighborhood of zero.

These results make use of the auxiliary polygon associated to a curve on a half-translation surface, as introduced by Tang and Webb.
\end{abstract}

\section{Introduction}

Let $S$ be a surface of genus $g$ with $p$ marked points and let $\mathrm{QD}(S)$ be the space of quadratic differentials on $S$. Each element $q \in \mathrm{QD}(S)$ naturally endows $S$ with a locally Euclidean metric with isolated conical singularities and linear holonomy restricted to $\{ \pm \mathrm{id}\}$. We also refer to elements of $\mathrm{QD}(S)$ as half-translation surfaces. There is a natural action of $\operatorname{SL}(2, \mathbb{R})$ on $\mathrm{QD}(S)$ preserving the signature of the singularities. A half-translation surface $q$ is called a Veech surface if its group of (derivatives of) affine self-diffeomorphisms is a lattice in $\mathrm{SL}(2, \mathbb{R})$. Veech surfaces possess remarkable dynamical properties akin to flat tori, and arise naturally in the contexts of rational billiards and Teichmüller curves in moduli space [Vee89].

In this paper, we study $\mathrm{QD}(S)$ from the point of view of simple closed curves on $S$. On a halftranslation surface $q$, a simple closed curve $\alpha$ either has a unique geodesic representative or there is a maximal flat cylinder on $q$ foliated by closed geodesics in the homotopy class of $\alpha$. In the former case, the geodesic representative $\alpha^{q}$ of $\alpha$ is a concatenation of saddle connections. We say that $\alpha$ is a crooked curve on $q$ if $\alpha^{q}$ has at least two saddle connections whose associated holonomy vectors are not parallel. We define the SL(2, R)-infimal length of $\alpha$ on $q$ to be

$$
l_{\alpha}^{\mathrm{SL}}(q)=\inf _{q^{\prime} \in \mathrm{SL}(2, \mathbb{R}) \cdot q} l_{\alpha}\left(q^{\prime}\right)
$$

where $l_{\alpha}(q)$ denotes the geodesic length of $\alpha$ on $q$. A curve $\alpha$ is crooked on $q$ if and only if $l_{\alpha}^{\mathrm{SL}}(q)$ is positive (see Proposition 2.1). Our first main result is a characterization of Veech surfaces in terms of their SL(2, R)-infimal length spectra.

Theorem 1.1. Let $q$ be a half-translation surface. Then $q$ is a Veech surface if and only if it has no short crooked curves: there is an $\epsilon>0$ such that $l_{\alpha}^{\mathrm{SL}}(q) \geq \epsilon$ for every crooked curve $\alpha$.

This result is reminiscent of the no-small-(virtual)-triangles theorem due to Smillie and Weiss [SW10]. They characterize Veech surfaces as the half-translation surfaces which possess a positive 
Max Forester, Robert Tang, and Jing Tao

lower bound on the areas of Euclidean triangles on $q$ with edges formed by saddle connections. One advantage of working with simple closed curves is that they are topological objects; they do not depend on the half-translation structure, and therefore we can study them at once over the entire quadratic differential space. In contrast, a saddle connection on $q$ persists only in an open subset of the relevant stratum of QD $(S)$.

One of the main tools we use in this paper is the auxiliary polygon $P_{\alpha}(q)$ associated to a simple closed curve $\alpha$ on $q$, as introduced by the second author and Webb in [TW15]. The area of $P_{\alpha}(q)$ gives an estimate for $l_{\alpha}^{\mathrm{SL}}(q)^{2}$ up to bounded multiplicative error (see Proposition 2.1(iii)). It follows that Theorem 1.1 is equivalent to the statement that $q$ is a Veech surface if and only if the polygonal area spectrum

$$
\operatorname{Poly}(q)=\left\{\operatorname{Area}\left(P_{\alpha}(q)\right): \alpha \text { is a simple closed curve }\right\}
$$

has a gap above zero. In fact, our arguments will prove a slightly stronger statement: either there is a gap (exactly when $q$ is a Veech surface), or this spectrum is dense in a neighborhood $[0, a)$ for some $a>0$. This statement also holds for the SL $(2, \mathbb{R})$-infimal length spectrum.

The forward implication of Theorem 1.1 will follow from a relatively straightforward application of the no-small-virtual-triangles theorem.

The reverse implication of Theorem 1.1 will make use of the auxiliary polygon mentioned above, as well as two other ingredients: the orbit closure theorem of Eskin, Mirzakhani, and Mohammadi [EMM15] and a rigidity statement for $\mathrm{SL}(2, \mathbb{R})$-orbits of quadratic differentials due to Duchin, Leininger, and Rafi [DLR10]. We remark that the orbit closure theorem is used only to deduce local path connectedness of $\mathrm{SL}(2, \mathbb{R})$-orbit closures in strata of half-translation surfaces.

To prove the reverse implication we use an elementary continuity argument; see Proposition 4.2. An essential step is establishing that the auxiliary polygon $P_{\alpha}(q)$ is continuous in $q$, with respect to the Hausdorff topology. This is achieved in several steps. By continuity of the intersection pairing between measured foliations on $S$, we obtain a continuous function $\mathrm{QD}^{1}(S) \rightarrow \mathscr{C}\left(\mathbb{R P} P^{1}, \mathbb{R}\right)$ of the

form $q \mapsto i\left(v_{q}^{\frac{\pi}{2}+\theta}, \alpha\right)$ for any fixed curve $\alpha$ on $S$. The value $i\left(v_{q}^{\frac{\pi}{2}+\theta}, \alpha\right)$ coincides with the width of the auxiliary polygon in direction $\theta$, and so the width function $w_{P_{\alpha}(q)} \in \mathscr{C}\left(\mathbb{R P} \mathrm{P}^{1}, \mathbb{R}\right)$ of the polygon is continuous in $q$. Finally, standard results from convex geometry on centrally symmetric sets yield continuity of $P_{\alpha}(q)$ itself.

As a further application of continuity of the auxiliary polygon, we show that $\operatorname{SL}(2, \mathbb{R})$-infimal length is continuous in $q$; see Proposition 3.12 .

Next consider the virtual triangle area spectrum, defined as follows:

$$
\operatorname{VT}(q)=\{|u \wedge v|: u, v \in \operatorname{hol}(q)\}
$$

where $\operatorname{hol}(q)$ is the set of holonomy vectors of saddle connections in $q$. The no-small-virtualtriangles theorem of [SW10] states that VT $(q)$ has a gap above zero if and only if $q$ is a Veech surface. In our second theorem we show further that $\operatorname{VT}(q)$ resembles the polygonal area spectrum as discussed above:

Theorem 1.2. Let $q$ be a half-translation surface. Then $V T(q)$ either has a gap above zero (exactly when $q$ is a Veech surface) or is dense in a neighborhood $[0, a)$ for some $a>0$. 
In fact, our argument provides a new proof of the "gap implies Veech" direction of the no-smallvirtual-triangles theorem. Moreover, the virtual triangles yielding the dense subset of $[0, a)$ in the non-Veech case are based virtual triangles; see Section 5 and Proposition 5.1. The non-Veech case of Theorem 1.2 is proved very similarly to Theorem 1.1.

Finally we have some additional results on polygonal area and $\operatorname{SL}(2, \mathbb{R})$-infimal length when $q$ is a Veech surface. The first of these will be derived from the analogous result for $\operatorname{VT}(q)$ in $[\mathrm{SW} 10]$.

Theorem 1.3. If $q$ is a Veech surface then $\operatorname{Poly}(q)$ is a discrete subset of $\mathbb{R}$.

Next, for $a>0$ define the sets

$$
\begin{aligned}
\operatorname{PA}(a) & =\left\{q \in \mathrm{QD}^{1}(S): \operatorname{Area}\left(\mathrm{P}_{\alpha}(q)\right) \geq a \text { for every crooked curve } \alpha \text { on } q\right\} \\
\operatorname{IL}(a) & =\left\{q \in \mathrm{QD}^{1}(S): l_{\alpha}^{\mathrm{SL}}(q) \geq a \text { for every crooked curve } \alpha \text { on } q\right\} .
\end{aligned}
$$

We say that two half-translation surfaces are affinely equivalent if they are related by the actions of the mapping class group and $\operatorname{SL}(2, \mathbb{R})$.

Theorem 1.4. For any $a>0$, the sets $P A(a)$ and $I L(a)$ both contain only finitely many affine equivalence classes of half-translation surfaces.

This result is an application of Theorem 2.2 of [EMM15], which classifies the closed SL(2, R)invariant subsets of strata of half-translation surfaces: namely, any such subset is a finite union of orbit closures.

\section{Acknowledgements}

The authors thank Alex Wright for many helpful conversations regarding $\mathrm{SL}(2, \mathbb{R})$-orbit closures. We also thank Jenya Sapir for encouraging us to prove continuity of $\operatorname{SL}(2, \mathbb{R})$-infimal length, and the referee for providing helpful feedback. Forester was partially supported by NSF award DMS1105765, and Tao by NSF award DMS-1311834.

\section{Background}

\subsection{Quadratic differentials and half-translation surfaces}

We begin by recalling relevant background regarding quadratic differentials and half-translation surfaces. For further details, consult [Str84].

Let $S$ be a surface of genus $g$ and let $\varrho \subset S$ be $p$ marked points with $3 g-3+p \geq 1$. A half-translation structure on $S$ consists of a finite set $\varsigma$ of singular points on $S$ (possibly including marked points), together with an atlas of charts to $\mathbb{C}$ defined away from $\varsigma$, where the transition maps are of the form $z \mapsto \pm z+c$ for some $c \in \mathbb{C}$. By pulling back the standard Euclidean metric on $\mathbb{C}$, one obtains a locally Euclidean metric on $S-\varsigma$. We require that the metric completion of this metric yields $S$ with a singular Euclidean structure, where every singularity has a cone angle $n \pi$ for some $n \geq 1$, and those that are not marked points must have cone angle at least $3 \pi$. The atlas determines a 
preferred vertical direction on $S$, and we consider this to be part of the data in the half-translation structure.

One can construct a half-translation surface by taking a finite collection of disjoint Euclidean polygons in $\mathbb{C}$, with pairs of edges identified by gluing maps of the form $z \mapsto \pm z+c$ [FM14].

By a quadratic differential $q$ on $S$, we mean a complex structure on $S$ equipped with an integrable holomorphic quadratic differential on $S-\varrho$ with finitely many zeros. This means that $q$ can extended to a meromorphic quadratic differential on $S$ with at worst simple poles at the marked points. The number of zeros minus the number of poles of $q$, counted with multiplicity, is $4 g-4$. The union of the set of zeros and poles will constitute the singularities of $q$. There is a natural holomorphic coordinate system $z$ on $S$ such that, in a neighborhood of a point away from a singularity, $q$ is given by $q=d z^{2}$. In a neighborhood of a zero of order $k \geq 1, q=z^{k} d z^{2}$, and in a neighborhood of a pole, $q=\frac{1}{z} d z^{2}$. A zero of order $k$ has cone angle $(k+2) \pi$ and a pole has cone angle $\pi$. Thus $q$ induces a half-translation structure on $S$, where the singular Euclidean metric is given locally by $|d z|^{2}$. We shall use $q$ to denote a quadratic differential on $S$, as well as $S$ equipped with the corresponding half-translation structure. The assumptions on $q$ ensure that the area of the half-translation surface, equal to $\int_{S}|q| d z^{2}$, is finite.

Let $\mathrm{QD}(S)$ be the space of quadratic differentials on $S$. This is a complex manifold of dimension $6 g-6+2 p$, and can be identified with the cotangent bundle to Teichmüller space $\mathscr{T}(S)$ via the natural projection $\mathrm{QD}(S) \rightarrow \mathscr{T}(S)$ obtained by taking the underlying complex structure of each half-translation surface. The space $\mathrm{QD}^{1}(S)$ of unit area quadratic differentials on $S$ can be identified with the unit cotangent bundle to $\mathscr{T}(S)$.

There is a natural $\operatorname{SL}(2, \mathbb{R})$-action on $\mathrm{QD}(S)$ defined by post-composing the coordinate charts to $\mathbb{C}$ by an $\mathbb{R}$-linear transformation. One can view this action by applying an element $A \in \operatorname{SL}(2, \mathbb{R})$ to a defining set of polygons for a half-translation surface $q$ to obtain a new half-translation structure $A \cdot q$, noting that parallel edges of the same length remain so under $\operatorname{SL}(2, \mathbb{R})$-deformations. Note that $\operatorname{SL}(2, \mathbb{R})$-deformations preserve area, and so $\operatorname{SL}(2, \mathbb{R})$ also acts naturally on $\mathrm{QD}^{1}(S)$.

We will write $e^{i \theta} \cdot q$ as shorthand for $\left(\begin{array}{cc}\cos \theta & -\sin \theta \\ \sin \theta & \cos \theta\end{array}\right) \cdot q$; or in natural coordinates at a regular point, $e^{i \theta} \cdot q=e^{i \theta} d z^{2}$

\subsection{Geodesic representatives and measured foliations}

Let $\mathscr{S}$ be the set of (free homotopy classes of) essential simple closed curves on $S$. For any $\alpha \in \mathscr{S}$, either $\alpha$ has a unique geodesic representative on $q$, or there is a unique maximal flat cylinder on $q$ foliated by the closed geodesics in the homotopy class of $\alpha$. In the former case, the geodesic representative of $\alpha$ is a concatenation of saddle connections - embedded geodesic arcs or loops with endpoints at singularities with no singularities on their interior. The angle between consecutive saddle connections is always at least $\pi$ on both sides. We shall use $\alpha^{q}$ to denote any geodesic representative of $\alpha$ on $q$.

If $\alpha^{q}$ is a core curve of a flat cylinder, then we call $\alpha$ a cylinder curve on $q$. Let cyl $(q)$ denote the set of cylinder curves on $q$, and $\widehat{c y l}(q)$ the set of curves whose geodesic representatives have constant direction on $q$. Any curve in $\mathscr{S}-\widehat{\operatorname{cyl}}(q)$ is called a crooked curve on $q$. 
Let $|d z|^{2}=d x^{2}+d y^{2}$ be the singular Euclidean metric associated to $q$. We can consider several notions of length of a curve $\alpha$ on $q$. The Euclidean length $l_{\alpha}(q)$ of $\alpha^{q}$ is given by integrating $\alpha^{q}$ with respect to $|d z|$ (in local coordinates). Integrating $\alpha^{q}$ with respect to $|d x|$ and $|d y|$ (in local coordinates) gives the horizontal and vertical lengths $l_{\alpha}^{H}(q)$ and $l_{\alpha}^{V}(q)$ respectively. Finally, define the SL(2, R)-infimal length of $\alpha$ with respect to $q$ to be

$$
l_{\alpha}^{\mathrm{SL}}(q)=\inf _{q^{\prime} \in \mathrm{SL}(2, \mathbb{R}) \cdot q} l_{\alpha}\left(q^{\prime}\right) .
$$

This should be viewed as a measure of length of $\alpha$ with respect to the $\operatorname{SL}(2, \mathbb{R})$-orbit of $q$, rather than with respect to $q$ itself.

Using the natural coordinate of $q$, one can pull back the foliation of $\mathbb{C}$ by lines in the direction $\theta \in \mathbb{R} \mathrm{P}^{1}$ to obtain a measured foliation $v_{q}^{\theta}$ on $q$, where the transverse measure is the Euclidean distance between leaves. In particular, the horizontal and vertical directions respectively give rise to the horizontal and vertical foliations $v_{q}^{H}=v_{q}^{0}$ and $v_{q}^{V}=v_{q}^{\pi / 2}$. The map $\mathrm{QD}(S) \times \mathbb{R P} \rightarrow \mathscr{M} \mathscr{F}(S)$ defined by $(q, \theta) \mapsto v_{q}^{\theta}$ is continuous, where $\mathscr{M} \mathscr{F}(S)$ is the space of measured foliations on $S$ [HM79]. Let $\mathscr{M} \mathscr{F}(q)=\left\{t \cdot v_{q}^{\theta}: \theta \in \mathbb{R P}^{1}, t \in \mathbb{R}_{+}\right\}$. Let $\mathscr{P} \mathscr{M} \mathscr{F}(S)$ and $\mathscr{P} \mathscr{M} \mathscr{F}(q)$ be the projectivizations of $\mathscr{M} \mathscr{F}(S)$ and $\mathscr{M} \mathscr{F}(q)$, respectively. Note that these sets are invariant under SL(2, $\mathbb{R})$-deformations.

The geometric intersection number $i: \mathscr{S} \times \mathscr{S} \rightarrow \mathbb{R}$ extends continuously to $\mathscr{M} \mathscr{F}(S) \times \mathscr{M} \mathscr{F}(S)$. For any curve $\alpha$, we have $i\left(v_{q}^{H}, \alpha\right)=l_{\alpha}^{V}(q)$ and $i\left(v_{q}^{V}, \alpha\right)=l_{\alpha}^{H}(q)$. See [FLP79] for additional background on measured foliations.

\subsection{Auxiliary polygons}

We now recall the construction from [TW15] of the auxiliary polygon $P_{\alpha}(q)$ associated to a curve $\alpha$ and a quadratic differential $q \in \mathrm{QD}(S)$.

To each saddle connection $e$ on $q$, we assign a holonomy vector $v_{e} \in \mathbb{R}^{2}$ which is parallel to it and is of the same length. This is well defined up to scaling by \pm 1 . For consistency, we require that the direction $\theta\left(v_{e}\right)$ (that is, the oriented angle from the positive $x$-axis to $v_{e}$ ) lies in the interval $[0, \pi)$. Consider a geodesic representative $\alpha^{q}$ on $q$. If $\alpha$ is a cylinder curve, we may choose $\alpha^{q}$ to be a boundary component of the maximal flat cylinder with core curve $\alpha$. Let $m_{e}$ be the number of times $\alpha^{q}$ runs over the saddle connection $e$, in either direction. Define

$$
P_{\alpha}(q)=\left\{\sum_{e} t_{e} m_{e} v_{e}:-\frac{1}{2} \leq t_{e} \leq \frac{1}{2}\right\} \subset \mathbb{R}^{2},
$$

where the sum is taken over all saddle connections used by $\alpha^{q}$.

The set $P_{\alpha}(q)$ is a convex Euclidean polygon, unless $\alpha^{q}$ has constant direction, in which case $P_{\alpha}(q)$ degenerates to a line segment parallel to $\alpha^{q}$ of length $l_{\alpha}(q)$. In particular, in the case of a cylinder curve, the definition of $P_{\alpha}(q)$ does not depend on the choice of boundary component. Moreover, this construction commutes with $\mathrm{SL}(2, \mathbb{R})$-deformations: for all $A \in \mathrm{SL}(2, \mathbb{R})$ we have $P_{\alpha}(A \cdot q)=$ $A \cdot P_{\alpha}(q)$.

Proposition 2.1 ([TW15]). Given a curve $\alpha \in \mathscr{S}$ and $q \in Q D^{1}(S)$, the auxiliary polygon $P_{\alpha}(q)$ defined above satisfies the following: 
(i) The perimeter of $P_{\alpha}(q)$ is $2 l_{\alpha}(q)$,

(ii) height $\left(P_{\alpha}(q)\right)=l_{\alpha}^{V}(q)$ and $\operatorname{width}\left(P_{\alpha}(q)\right)=l_{\alpha}^{H}(q)$,

(iii) $\pi \operatorname{Area}\left(P_{\alpha}(q)\right) \leq l_{\alpha}^{\mathrm{SL}}(q)^{2} \leq 8 \operatorname{Area}\left(P_{\alpha}(q)\right)$,

(iv) $\operatorname{Area}\left(P_{\alpha}(q)\right)=0$ if and only if $\alpha \in \widehat{\operatorname{cyl}}(q)$.

Here, the perimeter of a polygon $P$ is the length of its boundary $\partial P$. In the situation where $P$ degenerates to a line segment, we view $\partial P$ as a closed path traversing the line segment once in each direction.

The next property of $P_{\alpha}(q)$ that we need is really a statement about centrally symmetric convex polygons in general. Let $v_{1}, \ldots, v_{k}$ be non-zero vectors in $\mathbb{R}^{2}$ whose directions $\theta\left(v_{i}\right)$ satisfy

$$
0 \leq \theta\left(v_{1}\right) \leq \cdots \leq \theta\left(v_{k}\right)<\pi
$$

Let $P_{k}$ be the convex set $\left\{\sum_{i=1}^{k} t_{i} v_{i}:-1 / 2 \leq t_{i} \leq 1 / 2\right\}$.

Lemma 2.2. The points $p_{k}=\sum_{i=1}^{k} v_{i} / 2$ and $-p_{k}$ lie on the boundary $\partial P_{k}$. Furthermore,

(i) the boundary $\operatorname{arc} \partial^{+} P_{k}$, traveling counter-clockwise from $-p_{k}$ to $p_{k}$, is the path obtained by concatenating the vectors $v_{1}, \ldots, v_{k}$, in order. Similarly, the boundary arc $\partial^{-} P_{k}$ from $p_{k}$ to $-p_{k}$ is the concatenation of $-v_{1}, \ldots,-v_{k}$.

(ii) $P_{k}$ admits a tiling by the (possibly degenerate) parallelograms $v_{i} \times v_{j}$, with one copy of $v_{i} \times v_{j}$ for each unordered pair $i \neq j$.

Proof. We proceed by induction on $k$, the case $k=1$ being trivial (with $P_{1}$ a line segment).

Recall that the Minkowski sum of sets $A, B \subset \mathbb{R}^{2}$ is the set $A+B=\{a+b: a \in A, b \in B\}$. Observe that $P_{k}$ is the Minkowski sum of $P_{k-1}$ with the line segment $S_{k}$ with endpoints $\pm v_{k} / 2$. The description of $P_{k-1}$ given by the induction hypothesis allows us to see this sum clearly. The boundary of $P_{k}$ decomposes into four parts: the path $\partial^{+} P_{k-1}+\left\{-v_{k} / 2\right\}$, the path $\partial^{-} P_{k-1}+\left\{v_{k} / 2\right\}$, and two shifted copies of $S_{k}$; see Figure 1 . It is the assumption that $\theta\left(v_{k}\right)$ lies between $\theta\left(v_{i}\right)$ and $\pi$ for all $i$ that ensures that $\partial P_{k}$ is as described.
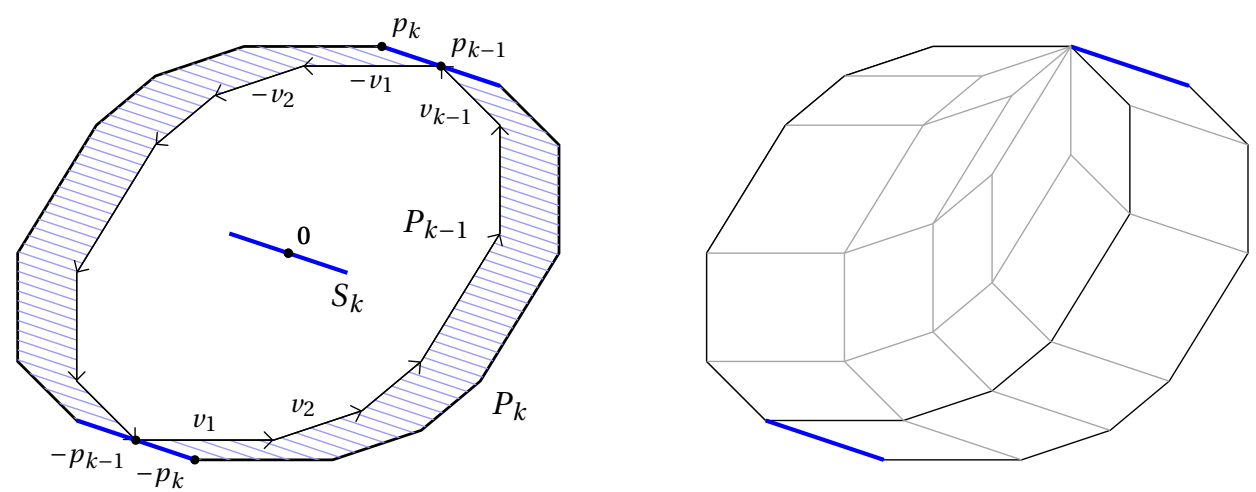

Figure 1: The polygon $P_{k}$ expressed as $P_{k-1}+S_{k}$, and tiled by parallelograms $v_{i} \times v_{j}$. 
The point $p_{k}$ is the initial point of the path $\partial^{-} P_{k-1}+\left\{v_{k} / 2\right\}$, which is on the boundary. Similarly, $-p_{k}$ is the initial point of $\partial^{+} P_{k-1}+\left\{-v_{k} / 2\right\}$, and conclusion (i) is now clear.

Next divide $P_{k}$ into two regions, one bounded by $\partial P_{k-1}+\left\{v_{k} / 2\right\}$, and the other bounded by the paths $\partial^{+} P_{k-1}+\left\{v_{k} / 2\right\}, \partial^{+} P_{k-1}+\left\{-v_{k} / 2\right\}$, and the two copies of $S_{k}$. The first region is isometric to $P_{k-1}$ and can be tiled by the parallelograms $v_{i} \times v_{j}, i<j<k$, by the induction hypothesis. The second region is the sum $\partial^{+} P_{k-1}+S_{k}$, and is tiled by the parallelograms $v_{i} \times v_{k}$, for all $i<k$, since $\partial^{+} P_{k-1}$ is a concatenation of the vectors $v_{i}, i<k$.

Since $P_{\alpha}(q)$ is $P_{k}$ for the vectors $\left\{m_{e} v_{e}: e\right.$ appears in $\left.\alpha\right\}$, ordered appropriately, we immediately deduce the following from Lemma 2.2(ii).

Lemma 2.3. For any $\alpha \in \mathscr{S}$ and $q \in Q D^{1}(S)$, we have

$$
\operatorname{Area}\left(P_{\alpha}(q)\right)=\sum_{e, e^{\prime}} m_{e} m_{e^{\prime}}\left|v_{e} \wedge v_{e^{\prime}}\right|
$$

where the sum is taken over all unordered pairs of distinct saddle connections $e, e^{\prime}$ appearing in $\alpha^{q}$.

\subsection{Rigidity}

A key tool that we use is the following result of Duchin, Leininger, and Rafi. Our formulation of the statement is slightly different from theirs, but their proof still works without modification.

Proposition 2.4 ([DLR10], Lemma 22). Let $q, q^{\prime} \in Q D^{1}(S)$ be half-translation surfaces. If $\widehat{c y l}(q) \subset$ $\widehat{\operatorname{cyl}}\left(q^{\prime}\right)$ then $\operatorname{SL}(2, \mathbb{R}) \cdot q=\operatorname{SL}(2, \mathbb{R}) \cdot q^{\prime}$.

Therefore, if $\operatorname{SL}(2, \mathbb{R}) \cdot q \neq \operatorname{SL}(2, \mathbb{R}) \cdot q^{\prime}$ then both $\widehat{\operatorname{cyl}}(q)-\widehat{\operatorname{cyl}}\left(q^{\prime}\right)$ and $\widehat{\operatorname{cyl}}\left(q^{\prime}\right)-\widehat{\operatorname{cyl}}(q)$ are non-empty.

Remark 2.5. One consequence of Proposition 2.4 is that when $3 g-3+p>1$, every $q \in \mathrm{QD}^{1}(S)$ has a crooked curve. This is true because $\mathrm{QD}^{1}(S)$ always has real dimension greater than 3 , and hence cannot be a single SL $(2, \mathbb{R})$-orbit.

Remark 2.6. Theorem 1 of [DLR10] states that the marked length spectrum of simple closed curves determines the half-translation surface $q$. Associated to $q$ is the marked polygonal area spectrum, which is the SL(2, $\mathbb{R})$-invariant function $\mathscr{S} \rightarrow \mathbb{R}$ given by $\alpha \mapsto \operatorname{Area}\left(P_{\alpha}(q)\right)$. We observe that, by Proposition 2.1(iv) and Proposition 2.4, the marked polygonal area spectrum of $q$ determines its $\operatorname{SL}(2, \mathbb{R})$-orbit.

\section{5 $\operatorname{SL}(2, \mathbb{R})$-orbit closures}

The space $\mathrm{QD}^{1}(S)$ of unit-area half translation structures on $S$ is naturally partitioned into strata $\mathrm{Q}(\kappa)$, where $\kappa$ is a partition of $4 g-4$ specifying the orders of the singularities. The mapping class group $\mathrm{MCG}(S)$ acts on $\mathrm{QD}^{1}(S)$ and each stratum $\mathrm{Q}(\kappa)$ by change of marking. The $\mathrm{SL}(2, \mathbb{R})-$ action on $\mathrm{QD}^{1}(S)$ preserves each stratum, and also descends naturally to the moduli space of half-translation surfaces $\operatorname{MQD}(S)=\mathrm{QD}^{1}(S) / \operatorname{MCG}(S)$, as well as each unmarked stratum $\mathrm{MQ}(\kappa)=$ $\mathrm{Q}(\kappa) / \mathrm{MCG}(S)$. Let $\pi: \mathrm{QD}^{1}(S) \rightarrow \mathrm{MQD}(S)$ be the natural projection, which is an orbifold covering 
map. Given $q \in \mathrm{Q}(\kappa)$, let $\mathrm{M}_{q}$ be the closure of $\pi(\mathrm{SL}(2, \mathbb{R}) \cdot q)$ in $\mathrm{MQ}(\kappa)$. We call $\mathrm{M}_{q}$ the orbit closure associated to $q$.

The structure of $\mathrm{M}_{q}$ has been elucidated in the work of Eskin, Mirzakhani, and Mohammadi, as follows:

Theorem 2.7 ([EMM15], Theorem 2.1). For any $q \in Q(\kappa)$, the orbit closure $\mathrm{M}_{q}$ is an affine invariant submanifold of $M Q(\kappa)$.

The statement given in [EMM15] actually refers to abelian differentials rather than quadratic differentials. However, the result applies equally well to the setting of quadratic differentials, by considering an appropriate two-fold branched covering of the surface.

The precise definition of "affine invariant submanifold" is rather involved and we shall not repeat it here in its entirety. It suffices to note that it includes the following:

- $\mathrm{M}_{q}$ is $\mathrm{SL}(2, \mathbb{R})$-invariant,

- $\mathrm{M}_{q}$ is the image of a properly immersed orbifold $f: N \rightarrow \mathrm{MQ}(\kappa)$.

Since $\pi: \mathrm{Q}(\kappa) \rightarrow \mathrm{MQ}(\kappa)$ is an orbifold covering, the preimage $\pi^{-1}\left(\mathrm{M}_{q}\right)$ is also the image of a properly immersed orbifold (in fact, manifold) of the same dimension as $N$.

The main conclusion we need to draw from Theorem 2.7 is that $\pi^{-1}\left(\mathrm{M}_{q}\right)$ is locally path connected. Let $\widetilde{\mathrm{M}}_{q}$ be the connected component of $\pi^{-1}\left(\mathrm{M}_{q}\right)$ containing $q$. Then local path connectedness of $\pi^{-1}\left(\mathrm{M}_{q}\right)$ implies that $\widetilde{\mathrm{M}}_{q}$ is open and closed in $\pi^{-1}\left(\mathrm{M}_{q}\right)$.

Now let $\Gamma_{q} \leq \operatorname{MCG}(S)$ be the (setwise) stabilizer of $\widetilde{\mathrm{M}}_{q}$, and define

$$
\mathscr{O}_{q}=\Gamma_{q} \cdot(\operatorname{SL}(2, \mathbb{R}) \cdot q) .
$$

Lemma 2.8. $\widetilde{\mathrm{M}}_{q}$ is the closure of $\mathscr{O}_{q}$ in $Q(\kappa)$.

Proof. Certainly, $\widetilde{\mathrm{M}}_{q}$ contains $\mathscr{O}_{q}$ and is closed in $\mathrm{Q}(\kappa)$, so it contains the closure of $\mathscr{O}_{q}$. Also, $\pi^{-1}\left(\mathrm{M}_{q}\right)$ is the closure of $\operatorname{MCG}(S) \cdot(\operatorname{SL}(2, \mathbb{R}) \cdot q)$. Note that if $g \in \operatorname{MCG}(S)-\Gamma_{q}$ then $g \cdot(\operatorname{SL}(2, \mathbb{R}) \cdot q)$ is contained in the component $g \widetilde{\mathrm{M}}_{q}$ of $\pi^{-1}\left(\mathrm{M}_{q}\right)$, which is disjoint from $\widetilde{\mathrm{M}}_{q}$. Now if $q^{\prime} \in \widetilde{\mathrm{M}}_{q}$ is a limit of a sequence of points $q_{i} \in g_{i} \cdot(\mathrm{SL}(2, \mathbb{R}) \cdot q)$, the open set $\widetilde{\mathrm{M}}_{q}$ must contain almost all $q_{i}$, and therefore $g_{i} \in \Gamma_{q}$ for almost all $i$, and $q^{\prime}$ is in the closure of $\mathscr{O}_{q}$.

We will also make use of the following finiteness result.

Theorem 2.9 ([EMM15], Theorem 2.2). Any closed $\mathrm{SL}(2, \mathbb{R})$-invariant subset of $Q(\kappa)$ is a finite union of $\mathrm{SL}(2, \mathbb{R})$-orbit closures.

\subsection{Veech surfaces}

Recall that $q \in \mathrm{QD}(S)$ is a Veech surface if its group of affine automorphisms is a lattice in SL(2, R). We now state several characterizations of Veech surfaces due to Smillie and Weiss [SW10], which builds on work of Vorobets [Vor96]. By a triangle on $q$, we mean a Euclidean triangle on $q$ with isometrically embedded interior, whose sides are saddle connections on $q$. Let hol $(q)$ be the set of holonomy vectors arising from saddle connections on $q$. 
Theorem 2.10 ([SW10]). For any $q \in Q(\kappa)$, the following are equivalent.

(i) $q$ is a Veech surface,

(ii) $q$ has no small triangles: there is a lower bound $\epsilon>0$ on the areas of all triangles on $q$,

(iii) $q$ has no small virtual triangles: there exists $\epsilon>0$ such that $|u \wedge \nu|>\epsilon$ for all pairs of nonparallel holonomy vectors $u, v \in \operatorname{hol}(q)$,

(iv) the virtual triangle area spectrum $\operatorname{VT}(q)=\{|u \wedge v|: u, v \in \operatorname{hol}(q)\}$ is discrete,

(v) $\pi(\mathrm{SL}(2, \mathbb{R}) \cdot q)$ is closed in $M Q(\kappa)$.

Note that condition (v) is the same as saying that $\mathrm{M}_{q}=\pi(\operatorname{SL}(2, \mathbb{R}) \cdot q)$. Applying Lemma 2.8, we deduce the following.

Corollary 2.11. A half-translation surface $q$ is a Veech surface if and only if $\widetilde{\mathrm{M}}_{q}=\mathscr{O}_{q}$.

\section{Continuity results}

\subsection{Continuity of the auxiliary polygon}

Here we show that the polygon $P_{\alpha}(q)$ is continuous in $q$, with respect to the Hausdorff topology in the plane. First we recall some basic notions from convex geometry. See, for instance, Sections 1.7 and 1.8 of [Sch14].

For any non-empty compact convex subset $K \subset \mathbb{R}^{2}$, the support function $h_{K}: S^{1} \rightarrow \mathbb{R}$ is defined by

$$
h_{K}(u)=\sup \{\langle x, u\rangle: x \in K\} .
$$

Here $\langle\cdot, \cdot\rangle$ is the usual inner product on $\mathbb{R}^{2}$. The width function $w_{K}: S^{1} \rightarrow \mathbb{R}$ is defined by

$$
w_{K}(u)=h_{K}(u)+h_{K}(-u) .
$$

Note that $w_{K}$ is even, and descends to a function on $\mathbb{R P} \mathrm{P}^{1}$ which we also denote by $w_{K}$. Now let $d_{H}$ denote Hausdorff distance. We have the following standard fact:

Lemma 3.1 ([Sch14],Lemma 1.8.14). Suppose $K$ and L are non-empty compact convex subsets of $\mathbb{R}^{2}$. Then

$$
d_{H}(K, L)=\sup _{u \in S^{1}}\left|h_{K}(u)-h_{L}(u)\right|
$$

Next consider centrally symmetric convex sets: these are convex sets $K$ such that $K=-K$. Note that the auxiliary polygons $P_{\alpha}(q)$ are both convex and centrally symmetric. If $K$ is centrally symmetric then $h_{K}(u)=h_{K}(-u)$ for all $u$, and therefore

$$
w_{K}=2 h_{K} .
$$

Now let $\|f\|$ denote the sup norm for functions $f: \mathbb{R P} \rightarrow \mathbb{R}$, and let $\mathscr{C}\left(\mathbb{R P} \mathrm{P}^{1}, \mathbb{R}\right)$ be the space of continuous functions, with the sup metric. Let $\mathscr{K}_{0}$ be the space of non-empty centrally symmetric compact convex sets in $\mathbb{R}^{2}$, with the Hausdorff metric. The next lemma follows directly from Lemma 3.1 and equation (3.2). 
Lemma 3.3. If $K, L \in \mathscr{K}_{0}$ then

$$
2 d_{H}(K, L)=\sup _{\theta \in \mathbb{R P}^{1}}\left|w_{K}(\theta)-w_{L}(\theta)\right|=\left\|w_{K}-w_{L}\right\| .
$$

Corollary 3.4. The map $W: \mathscr{K}_{0} \rightarrow \mathscr{C}\left(\mathbb{R P} \mathrm{P}^{1}, \mathbb{R}\right)$ given by $K \mapsto \frac{1}{2} w_{K}$ is an isometric embedding.

Let us now return our attention to the auxiliary polygons.

Theorem 3.5. The map $Q D^{1}(S) \times \mathscr{S} \rightarrow \mathscr{K}_{0}$ defined by $(q, \alpha) \mapsto P_{\alpha}(q)$ is continuous in the first factor.

Proof. Let $\alpha \in \mathscr{S}$ be fixed. Applying Proposition 2.1, we see that

$$
w_{P_{\alpha}(q)}(\theta)=\operatorname{width}\left(e^{-i \theta} \cdot P_{\alpha}(q)\right)=l_{\alpha}^{H}\left(e^{-i \theta} \cdot q\right)=i\left(v_{q}^{\frac{\pi}{2}+\theta}, \alpha\right)
$$

for all $q \in \mathrm{QD}^{1}(S)$ and $\theta \in \mathbb{R} \mathrm{P}^{1}$. The map $\mathrm{QD}^{1}(S) \times \mathbb{R P} \mathrm{P}^{1} \rightarrow \mathcal{M} \mathscr{F}(S)$ given by $(q, \theta) \mapsto v_{q}^{\frac{\pi}{2}+\theta}$ is continuous. By continuity of intersection number on $\mathscr{M} \mathscr{F}(S) \times \mathscr{M} \mathscr{F}(S)$ and compactness of $\mathbb{R} \mathrm{P}^{1}$, the map $q \mapsto w_{P_{\alpha}(q)}$ defines a continuous function from $\mathrm{QD}^{1}(S)$ to $\mathscr{C}\left(\mathbb{R P}^{1}, \mathbb{R}\right)$. Moreover, its image is contained in $W\left(\mathscr{K}_{0}\right)$, and composing this map with $\frac{1}{2} W^{-1}$ yields the function $q \mapsto P_{\alpha}(q)$. This map is continuous by Corollary 3.4.

Finally, applying continuity of Area: $\mathscr{K}_{0} \rightarrow \mathbb{R}[\operatorname{Sch} 14$, Theorem 1.8.20] yields the desired result.

Corollary 3.6. The function Area: $Q D^{1}(S) \times \mathscr{S} \rightarrow \mathbb{R}_{\geq 0}$ defined by $\operatorname{Area}(q, \alpha)=\operatorname{Area}\left(P_{\alpha}(q)\right)$ is continuous and $\mathrm{SL}(2, \mathbb{R})$-invariant in the first factor.

\subsection{Continuity of $\operatorname{SL}(2, \mathbb{R})$-infimal length}

In this section, we apply continuity of the auxiliary polygon to deduce continuity of $\operatorname{SL}(2, \mathbb{R})-$ infimal length.

Given $K \in \mathscr{K}_{0}$, let $r^{-}(K)=\inf _{u \in S^{1}} h_{K}(u)$ and $r^{+}(K)=\sup _{u \in S^{1}} h_{K}(u)$. One can show that these two numbers coincide with the minimum and maximum distances to the origin of points on $\partial K$. Define the eccentricity of $K$ to be $\operatorname{ecc}(K)=\frac{r^{+}(K)}{r^{-}(K)} \geq 1$. Note that $r^{-}(K)=0$ if and only if $K$ is degenerate. Recall that any matrix $A \in \operatorname{SL}(2, \mathbb{R})$ has a singular value decomposition

$$
A=e^{i \theta_{1}}\left(\begin{array}{cc}
\lambda & 0 \\
0 & \lambda^{-1}
\end{array}\right) e^{i \theta_{2}}
$$

for some stretch factor $\lambda \geq 1$ and $\theta_{1}, \theta_{2} \in \mathbb{R}$. Moreover, $\lambda=\lambda(A)$ is unique, and $\lambda(A B) \leq \lambda(A) \lambda(B)$ and $\lambda\left(A^{-1}\right)=\lambda(A)$ for all $A, B \in \mathrm{SL}(2, \mathbb{R})$. Also note that

$$
\lambda(A) r^{-}(K) \leq r^{+}(A \cdot K) \leq \lambda(A) r^{+}(K) \text { and } \frac{r^{-}(K)}{\lambda(A)} \leq r^{-}(A \cdot K) \leq \frac{r^{+}(K)}{\lambda(A)}
$$

for all $K \in \mathscr{K}_{0}$. Since $A$ acts as a $\lambda(A)$-Lipschitz map from $\mathbb{R}^{2}$ to itself, we deduce for all $K, L \in \mathscr{K}_{0}$ that

$$
d_{H}(A \cdot K, A \cdot L) \leq \lambda(A) d_{H}(K, L)
$$


Lemma 3.9. Fix $r_{0}>0$, and suppose $K, L \in \mathscr{K}_{0}$ satisfy $r^{-}(K), r^{-}(L)>r_{0}$. Then

$$
\left(1+\frac{d_{H}(K, L)}{r_{0}}\right)^{-2}<\frac{\mathrm{ecc}(K)}{\operatorname{ecc}(L)}<\left(1+\frac{d_{H}(K, L)}{r_{0}}\right)^{2} .
$$

Proof. Let $D=d_{H}(K, L)$. Applying Lemma 3.1 gives

$$
\frac{\operatorname{ecc}(K)}{\operatorname{ecc}(L)}=\frac{r^{+}(K)}{r^{+}(L)} \frac{r^{-}(L)}{r^{-}(K)} \leq\left(\frac{r^{+}(L)+D}{r^{+}(L)}\right)\left(\frac{r^{-}(K)+D}{r^{-}(K)}\right)<\left(1+\frac{D}{r_{0}}\right)^{2} .
$$

The other bound can be deduced similarly.

Define the eccentricity of a curve $\alpha \in \mathscr{S}$ on $q \in \mathrm{QD}^{1}(S)$ to be $\operatorname{ecc}_{\alpha}(q)=\operatorname{ecc}\left(P_{\alpha}(q)\right)$. Note that $\operatorname{ecc}_{\alpha}(q)=\infty$ if and only if $\alpha \in \widehat{\operatorname{cyl}}(q)$. By Theorem 3.5, ecc $\alpha$ is continuous on the set of quadratic differentials on which $\alpha$ is crooked.

Lemma 3.10. Let $\alpha$ be a crooked curve on $q \in Q D^{1}(S)$, and suppose the $\operatorname{SL}(2, \mathbb{R})$-infimal length of $\alpha$ is attained at $m \in \mathrm{SL}(2, \mathbb{R}) \cdot q$. There is a constant $c \geq 1$ (independent of $S, q$, and $\alpha$ ) such that

$$
\frac{\lambda(A)^{2}}{c} \leq \operatorname{ecc}_{\alpha}(A \cdot m) \leq c \lambda(A)^{2}
$$

for all $A \in \mathrm{SL}(2, \mathbb{R})$. In particular, $\operatorname{ecc}_{\alpha}(m) \leq c$.

Proof. By Lemma 6.4 and Corollary 7.2 of [TW15], there exists $A_{0} \in \mathrm{SL}(2, \mathbb{R})$ such that $\operatorname{ecc}_{\alpha}\left(A_{0} \cdot m\right) \leq$ 2 and $\lambda\left(A_{0}\right)<c^{\prime}$ for a constant $c^{\prime}$ independent of $S, q$, and $\alpha$. Taking $c \geq 2\left(c^{\prime}\right)^{2}$, the result follows by applying the inequalities in (3.7), with $K=P_{\alpha}\left(A_{0} \cdot m\right)$.

Lemma 3.11. Let $\alpha$ be a crooked curve on $q \in Q D^{1}(S)$. Then for all $\epsilon>0$ and $\lambda_{0} \geq 1$, there exists an open neighborhood $U \subseteq Q D^{1}(S)$ of $q$ such that for all $q^{\prime} \in U$ and $A \in \mathrm{SL}(2, \mathbb{R})$ satisfying $\lambda(A) \leq \lambda_{0}$, we have:

(i) $(1+\epsilon)^{-2}<\frac{\operatorname{ecc}_{\alpha}\left(A \cdot q^{\prime}\right)}{\operatorname{ecc}_{\alpha}(A \cdot q)}<(1+\epsilon)^{2}$,

(ii) $\left|l_{\alpha}(A \cdot q)-l_{\alpha}\left(A \cdot q^{\prime}\right)\right|<\pi \epsilon$.

Proof. Set $r_{0}=\frac{r^{-}\left(P_{\alpha}(q)\right)}{2 \lambda_{0}}$, and choose $\delta<\min \left\{\frac{r^{-}\left(P_{\alpha}(q)\right)}{2}, \frac{r_{0} \epsilon}{\lambda_{0}}, \frac{\epsilon}{\lambda_{0}}\right\}$.

By Theorem 3.5, there is an open neighborhood $U$ of $q$ with $d_{H}\left(P_{\alpha}(q), P_{\alpha}\left(q^{\prime}\right)\right)<\delta$ for all $q^{\prime} \in U$. Choose any $q^{\prime} \in U$ and $A \in \mathrm{SL}(2, \mathbb{R})$ satisfying $\lambda(A) \leq \lambda_{0}$. Then

$$
d_{H}\left(P_{\alpha}(A \cdot q), P_{\alpha}\left(A \cdot q^{\prime}\right)\right)<\min \left\{r_{0} \epsilon, \epsilon\right\}
$$

by (3.8). Now by (3.7), we deduce

$$
r^{-}\left(P_{\alpha}\left(A \cdot q^{\prime}\right)\right) \geq \frac{r^{-}\left(P_{\alpha}\left(q^{\prime}\right)\right)}{\lambda(A)}>\frac{r^{-}\left(P_{\alpha}(q)\right)-\delta}{\lambda_{0}}>\frac{r^{-}\left(P_{\alpha}(q)\right)}{2 \lambda_{0}}=r_{0}
$$


Applying Lemma 3.9 yields the first claim. For the second claim, we use the well-known fact that the perimeter of any convex region $K \subset \mathbb{R}^{2}$ is $\int_{0}^{\pi} w_{K}(u) d u$, where $w_{K}(u)$ is the width of $K$ at $u \in S^{1}$. Set $w_{q}(u)$ to be the width of $P_{\alpha}(q)$ at $u$. Then using Proposition 2.1(i) and Lemma 3.3 we have

$$
\begin{aligned}
\left|l_{\alpha}(A \cdot q)-l_{\alpha}\left(A \cdot q^{\prime}\right)\right| & =\frac{1}{2}\left|\int_{0}^{\pi} w_{A \cdot q}(u)-w_{A \cdot q^{\prime}}(u) d u\right| \\
& \leq \frac{1}{2} \int_{0}^{\pi}\left|w_{A \cdot q}(u)-w_{A \cdot q^{\prime}}(u)\right| d u \\
& \leq \pi d_{H}\left(P_{\alpha}(A \cdot q), P_{\alpha}\left(A \cdot q^{\prime}\right)\right)<\pi \epsilon .
\end{aligned}
$$

Proposition 3.12. For any curve $\alpha \in \mathscr{S}$, the function $l_{\alpha}^{\mathrm{SL}}: Q D^{1}(S) \rightarrow \mathbb{R}_{\geq 0}$ is continuous and $\mathrm{SL}(2, \mathbb{R})$ invariant.

Proof. Fix $\alpha \in \mathscr{S}$ and $q \in \mathrm{QD}^{1}(S)$. We now prove that $l_{\alpha}^{\mathrm{SL}}$ is continuous at $q$. In the case where $\alpha \in \widehat{\operatorname{cyl}}(q)$, we have $l_{\alpha}^{\mathrm{SL}}(q)=0=\operatorname{Area}(q, \alpha)$. Continuity of $l_{\alpha}^{\mathrm{SL}}$ at $q$ then follows from Proposition 2.1(iii) and Corollary 3.6.

Assume $\alpha$ is crooked on $q$. Given any $\epsilon \in(0,1)$, we shall show that $\left|l_{\alpha}^{\mathrm{SL}}(q)-l_{\alpha}^{\mathrm{SL}}\left(q^{\prime}\right)\right|<\pi \epsilon$ for all $q^{\prime}$ in a sufficiently small neighborhood of $q$. Suppose $l_{\alpha}^{\mathrm{SL}}(q)$ is attained at $A_{1} \cdot q$, for some $A_{1} \in \operatorname{SL}(2, \mathbb{R})$. Choose $\lambda_{0} \geq 2 c \lambda\left(A_{1}\right)$, where $c$ is the constant from Lemma 3.10. Let $U$ be an open neighborhood of $q$ which satisfies the conclusion of Lemma 3.11. For $q^{\prime} \in U$, let $A_{2} \in \operatorname{SL}(2, \mathbb{R})$ be such that $l_{\alpha}^{\mathrm{SL}}\left(q^{\prime}\right)=$ $l_{\alpha}\left(A_{2} \cdot q^{\prime}\right)$. By Lemmas 3.10 and $3.11(\mathrm{i})$, we have

$$
\operatorname{ecc}_{\alpha}\left(A_{1} \cdot q^{\prime}\right)<(1+\epsilon)^{2} \operatorname{ecc}_{\alpha}\left(A_{1} \cdot q\right)<(1+\epsilon)^{2} c<4 c .
$$

Applying Lemma 3.10 with $m=A_{2} \cdot q^{\prime}$, we obtain $\lambda\left(A_{1} A_{2}^{-1}\right)^{2} \leq c \cdot \operatorname{ecc}_{\alpha}\left(A_{1} \cdot q^{\prime}\right)$, and therefore $\lambda\left(A_{1} A_{2}^{-1}\right) \leq 2 c$. Thus,

$$
\lambda\left(A_{2}\right) \leq \lambda\left(A_{2} A_{1}^{-1}\right) \lambda\left(A_{1}\right)=\lambda\left(A_{1} A_{2}^{-1}\right) \lambda\left(A_{1}\right) \leq 2 c \lambda\left(A_{1}\right) \leq \lambda_{0} .
$$

Finally, applying Lemma 3.11(ii) yields

$$
\begin{aligned}
l_{\alpha}^{\mathrm{SL}}(q)-\pi \epsilon \leq l_{\alpha}\left(A_{2} \cdot q\right)-\pi \epsilon<l_{\alpha}\left(A_{2} \cdot q^{\prime}\right)=l_{\alpha}^{\mathrm{SL}}\left(q^{\prime}\right) & \\
& \leq l_{\alpha}\left(A_{1} \cdot q^{\prime}\right)<l_{\alpha}\left(A_{1} \cdot q\right)+\pi \epsilon=l_{\alpha}^{\mathrm{SL}}(q)+\pi \epsilon
\end{aligned}
$$

as required.

\section{The polygonal area spectrum}

We are now ready to prove Theorem 1.1. The first step is Theorem 1.3, which says that Poly $(q)$ is discrete if $q$ is a Veech surface.

Proof of Theorem 1.3. By Theorem 2.10(iv), the virtual triangle area spectrum VT( $q$ ) is discrete. For each simple closed curve $\alpha$, $\operatorname{Area}\left(P_{\alpha}(q)\right)$ is a positive integer combination of numbers from the set $\operatorname{VT}(q)$, by Lemma 2.3. The result follows.

Using Proposition 2.1(iii), we obtain: 
Corollary 4.1. If $q \in Q D^{1}(S)$ is a Veech surface then

$$
\inf \left\{l_{\alpha}^{\mathrm{SL}}(q): \alpha \text { is crooked on } q\right\}>0
$$

For the converse, it is worth remarking that the existence of short crooked curves is not an immediately obvious consequence of having small virtual triangles. From a given collection of saddle connections on $q$ for which $|u \wedge v|$ can be taken to be arbitrarily small, it appears difficult to construct a sequence of saddle connections to satisfy the following:

- no two saddle connections intersect (in their interiors),

- consecutive saddle connections meet with an angle of at least $\pi$ on both sides,

- their concatenation is homotopic to an essential simple closed curve.

(Small triangles on $q$ are not particularly useful since their sides must meet at an angle of less than $\pi$.) In our proof below, the auxiliary polygon plays a key role in bypassing this difficulty.

For $q \in \mathrm{Q}(\kappa)$, recall that $\mathscr{O}_{q}=\Gamma_{q} \cdot(\mathrm{SL}(2, \mathbb{R}) \cdot q)$ is a dense subset of $\widetilde{\mathrm{M}}_{q}$ in $\mathrm{Q}(\kappa)$, where $\Gamma_{q} \leq \mathrm{MCG}(S)$ is the stabilizer of $\widetilde{M}_{q}$.

Proposition 4.2. Suppose $q \in Q D^{1}(S)$ is not a Veech surface. Then there is a number a $>0$ such that the polygonal area spectrum $\operatorname{Poly}(q)$ contains a dense subset of $[0, a]$.

Proof. Applying Corollary 2.11, we have $\widetilde{\mathrm{M}}_{q} \neq \mathscr{O}_{q}$ and so we may choose $q^{\prime} \in \widetilde{\mathrm{M}}_{q}-\mathscr{O}_{q}$. Then $\mathrm{SL}(2, \mathbb{R}) \cdot q \neq \mathrm{SL}(2, \mathbb{R}) \cdot q^{\prime}$, and so by Proposition 2.4 , there exists a curve $\alpha \in \widehat{\operatorname{cyl}}(q)-\widehat{\operatorname{cyl}}\left(q^{\prime}\right)$. By Proposition 2.1(iv), we have $\operatorname{Area}(q, \alpha)=0$ and $\operatorname{Area}\left(q^{\prime}, \alpha\right)=a>0$. Since $\widetilde{\mathrm{M}}_{q}$ is connected and $\operatorname{Area}(\cdot, \alpha)$ is continuous, by Corollary 3.6, we deduce that $[0, a] \subseteq \operatorname{Area}\left(\widetilde{\mathrm{M}}_{q}, \alpha\right)$. It follows that $\operatorname{Area}\left(\mathscr{O}_{q}, \alpha\right)$, and hence $\operatorname{Area}\left(\mathscr{O}_{q}, \mathscr{S}\right)$, contains a dense subset of $[0, a]$. Finally, the polygonal area spectrum $\operatorname{Poly}(q)=\operatorname{Area}(q, \mathscr{S})$ is invariant under $\mathrm{SL}(2, \mathbb{R})$-deformations and changes of markings, and therefore $\operatorname{Area}(q, \mathscr{S})=\operatorname{Area}\left(\mathscr{O}_{q}, \mathscr{S}\right)$.

Note that this proof uses only the fact that $\operatorname{Area}(\cdot, \alpha)$ is a continuous $\operatorname{SL}(2, \mathbb{R})$-invariant function to $\mathbb{R}_{\geq 0}$ which takes the value 0 precisely when $\alpha \in \widehat{\operatorname{cyl}}(q)$. Since the same properties hold for $l_{\alpha}^{\mathrm{SL}}$, we may argue as above to deduce:

Proposition 4.3. If $q \in Q D^{1}(S)$ is not a Veech surface then there exists $a>0$ such that the $\operatorname{SL}(2, \mathbb{R})-$ infimal length spectrum $\left\{l_{\alpha}^{\mathrm{SL}}(q): \alpha \in \mathscr{S}\right\}$ contains a dense subset of $[0, a]$.

We conclude this section with a proof of Theorem 1.4, which is restated below. For $a>0$, recall that

$$
\begin{aligned}
\mathrm{PA}(a) & =\left\{q \in \mathrm{QD}^{1}(S): \operatorname{Area}\left(\mathrm{P}_{\alpha}(q)\right) \geq a \text { for every crooked curve } \alpha \text { on } q\right\} \\
\mathrm{IL}(a) & =\left\{q \in \mathrm{QD}^{1}(S): l_{\alpha}^{\mathrm{SL}}(q) \geq a \text { for every crooked curve } \alpha \text { on } q\right\} .
\end{aligned}
$$

Theorem 4.4. For any $a>0$, the sets $P A(a)$ and $I L(a)$ both contain only finitely many affine equivalence classes of half-translation surfaces. 
Proof. Applying Corollary 3.6, we deduce that $\mathrm{PA}(a)$ is a closed and SL(2, $\mathbb{R})$-invariant subset of $\mathrm{QD}^{1}(S)$. Moreover, $\mathrm{PA}(a)$ is invariant under the action of $\operatorname{MCG}(S)$, and so $\mathrm{PA}(a)$ descends to a closed $\mathrm{SL}(2, \mathbb{R})$-invariant subset $C$ in $\operatorname{MQD}(S)$. It follows that $C \cap \mathrm{Q}(\kappa)$ is closed and $\mathrm{SL}(2, \mathbb{R})-$ invariant in each stratum $\mathrm{Q}(\kappa)$ under the subspace topology. By Theorem 2.9, $C \cap \mathrm{Q}(\kappa)$ is a finite union of SL $(2, \mathbb{R})$-orbit closures. Since elements of $\operatorname{PA}(a)$ are necessarily Veech surfaces by Theorem 1.1, $C \cap \mathrm{Q}(\kappa)$ must be a finite union of $\mathrm{SL}(2, \mathbb{R})$-orbits. Finally, there are only finitely many strata for a given genus, and so the desired result follows.

The proof for IL $(a)$ proceeds identically using Proposition 3.12 in place of Corollary 3.6.

\section{The virtual triangle area spectrum}

We are almost ready to prove Theorem 1.2. In the introduction we defined the virtual triangle area spectrum $\operatorname{VT}(q)$. Let $\operatorname{VT}_{0}(q) \subset \operatorname{VT}(q)$ be the subset consisting of the numbers $|u \wedge v|$ such that $u$ and $v$ are the holonomy vectors of a pair of saddle connections with a common endpoint (that is, a based virtual triangle). Note that saddle connections forming a based virtual triangle need not form a triangle, since they may have angle $\pi$ or more on both sides. We also define $\mathrm{VT}_{0}(X)=\bigcup_{q \in X} \mathrm{VT}_{0}(q)$ for any set $X \subset \mathrm{QD}^{1}(S)$.

We know from the implication (i) $\Rightarrow$ (iii) of Theorem 2.10 that if $q$ is a Veech surface then $\operatorname{VT}(q)$ has a gap above zero. The remainder of Theorem 1.2 follows from the next proposition:

Proposition 5.1. Suppose $q \in Q D^{1}(S)$ is not a Veech surface. Then there is a number $a>0$ such that $V T_{0}(q)$ contains a dense subset of $[0, a]$.

Proof. As in the proof of Proposition 4.2, there exist a half-translation surface $q^{\prime} \in \widetilde{\mathrm{M}}_{q}-\mathscr{O}_{q}$ and a curve $\alpha \in \widehat{\operatorname{cyl}}(q)-\widehat{\operatorname{cyl}}\left(q^{\prime}\right)$, and we know that $\operatorname{Area}(q, \alpha)=0$ and $\operatorname{Area}\left(q^{\prime}, \alpha\right)>0$. Let $q_{t}$ be a path in $\widetilde{\mathrm{M}}_{q}$ from $q_{0}=q$ to $q_{1}=q^{\prime}$. Consider the set

$$
\left\{t \in[0,1]: \operatorname{Area}\left(q_{t}, \alpha\right)>0\right\},
$$

which is an open neighborhood of 1 in $[0,1]$ that does not contain 0 . It has a connected component $\left(t_{0}, 1\right]$. Replacing the path $q_{t}$ by its restriction to $\left[t_{0}, 1\right]$ and reparametrizing over $[0,1]$, we have $\operatorname{Area}\left(q_{0}, \alpha\right)=0$ and $\operatorname{Area}\left(q_{t}, \alpha\right)>0$ for all $t \in(0,1]$.

Now consider the geodesic representative $\alpha^{q_{0}}$ and express it as a concatenation of saddle connections $e_{1} \cdots e_{k}$. Let $\alpha_{i}$ be the topological arc represented by $e_{i}$; it is an isotopy class rel endpoints, where the interior of the arc is required to avoid the singularities. Consider the set

$$
\left\{t \in[0,1]: \text { each } \alpha_{i} \text { is represented by a saddle connection in } q_{t}\right\} \text {. }
$$

This set is an open neighborhood of 0 in $[0,1]$. Again, replacing the path $q_{t}$ by its restriction to an interval $[0, \epsilon]$ and reparametrizing over $[0,1]$, we may assume that the arcs $\alpha_{1}, \ldots, \alpha_{k}$ are represented by saddle connections for all $t \in[0,1]$. Let $e_{i}(t)$ denote the saddle connection in $q_{t}$ representing $\alpha_{i}$. Let $v_{i}(t)$ be the holonomy vector of $e_{i}(t)$.

Define functions $\phi_{i}(t)=\left|v_{i}(t) \wedge v_{i+1}(t)\right|$ for each $i$ (with indices taken mod $k$ ). These are continuous because holonomy vectors vary continuously where defined. Since all $e_{i}$ are parallel on $q_{0}$, 
we have $\phi_{i}(0)=0$ for all $i$. We claim that $\phi_{i}\left(t_{1}\right)>0$ for some $t_{1}>0$ and some $i$. If not, then for all $t \in[0,1]$ the saddle connections $e_{i}(t)$ are all parallel. The angles between consecutive saddle connections (on either side) must remain constant, since they are constrained to lie in the discrete set $\pi \mathbb{Z}$, and therefore the concatenation $e_{1}(t) \cdots e_{k}(t)$ remains a geodesic representative for $\alpha$ on $q_{t}$. But this contradicts the fact that $\operatorname{Area}\left(q_{t}, \alpha\right)>0$ for all $t \in(0,1]$.

Restricting $q_{t}$ to $\left[0, t_{1}\right]$ and reparametrizing over $[0,1]$ one last time, we have a path $q_{t}$ in $\widetilde{\mathrm{M}}_{q}$ and a pair of saddle connections $e_{i}(t), e_{i+1}(t)$ which persist on $q_{t}$ throughout the path, such that $\phi_{i}(0)=$ $\left|v_{i}(0) \wedge v_{i+1}(0)\right|=0$ and $\phi_{i}(1)=\left|v_{i}(1) \wedge v_{i+1}(1)\right|=a>0$. The function $\phi_{i}$ is defined and continuous on the open set $U \subset \widetilde{\mathrm{M}}_{q}$ where $e_{i}$ and $e_{i+1}$ persist. This set contains the path $q_{t}$ and hence $\phi_{i}(U)$ contains $[0, a]$. Since $U \cap \mathscr{O}_{q}$ is dense in $U$, it follows that $\phi_{i}\left(U \cap \mathscr{O}_{q}\right)$, and hence $\operatorname{VT}_{0}\left(\mathscr{O}_{q}\right)$, contains a dense subset of $[0, a]$. Finally, the based virtual triangle area spectrum is invariant under change of marking and $\operatorname{SL}(2, \mathbb{R})$, and so $\operatorname{VT}_{0}(q)=\operatorname{VT}_{0}\left(\mathscr{O}_{q}\right)$.

\section{References}

[DLR10] Moon Duchin, Christopher J. Leininger, and Kasra Rafi, Length spectra and degeneration of flat metrics, Invent. Math. 182 (2010), no. 2, 231-277. MR 2729268 (2011m:57022)

[EMM15] Alex Eskin, Maryam Mirzakhani, and Amir Mohammadi, Isolation, equidistribution, and orbit closures for the $\mathrm{SL}(2, \mathbb{R})$ action on moduli space, Ann. of Math. (2) 182 (2015), no. 2, 673-721. MR 3418528

[FLP79] Albert Fathi, François Laudenbach, and Valentin Poénaru, Travaux de Thurston sur les surfaces, Astérisque, vol. 66, Société Mathématique de France, Paris, 1979, Séminaire Orsay, With an English summary. MR 568308

[FM14] Giovanni Forni and Carlos Matheus, Introduction to Teichmüller theory and its applications to dynamics of interval exchange transformations, flows on surfaces and billiards, J. Mod. Dyn. 8 (2014), no. 3-4, 271-436. MR 3345837

[HM79] John Hubbard and Howard Masur, Quadratic differentials and foliations, Acta Math. 142 (1979), no. 3-4, 221-274. MR 523212

[Sch14] Rolf Schneider, Convex bodies: the Brunn-Minkowski theory, expanded ed., Encyclopedia of Mathematics and its Applications, vol. 151, Cambridge University Press, Cambridge, 2014. MR 3155183

[Str84] Kurt Strebel, Quadratic differentials, Ergebnisse der Mathematik und ihrer Grenzgebiete (3) [Results in Mathematics and Related Areas (3)], vol. 5, Springer-Verlag, Berlin, 1984. MR 743423

[SW10] John Smillie and Barak Weiss, Characterizations oflattice surfaces, Invent. Math. 180 (2010), no. 3, 535-557. MR 2609249 (2012c:37072)

[TW15] Robert Tang and Richard C. H. Webb, Shadows of Teichmüller discs in the curve graph, 2015 preprint, to appear in Int. Math. Res. Not. IMRN.

[Vee89] W. A. Veech, Teichmüller curves in moduli space, Eisenstein series and an application to triangular billiards, Invent. Math. 97 (1989), no. 3, 553-583. MR 1005006

[Vor96] Ya. B. Vorobets, Planar structures and billiards in rational polygons: the Veech alternative, Uspekhi Mat. Nauk 51 (1996), no. 5(311), 3-42. MR 1436653 (97j:58092) 
Mathematics Department, University of Oklahoma, Norman, OK 73019, USA

forester@math.ou.edu, rtang@math.ou.edu, jing@math.ou.edu 